\title{
La part « ottomane » dans les pratiques musicales des Grecs de Smyrne
}

The "Ottoman" part in Smyrnaian Greek musical practice

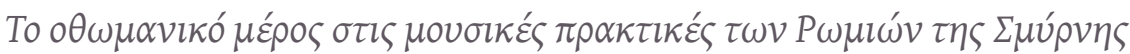

\section{Basma Zerouali}

\section{(2) OpenEdition}

\section{Journals}

\section{Édition électronique}

URL : https://journals.openedition.org/ceb/4518

DOI : $10.4000 /$ ceb.4518

ISSN : 2261-4184

Éditeur

INALCO

Édition imprimée

Date de publication : 1 janvier 2004

ISSN : 0290-7402

Référence électronique

Basma Zerouali, « La part « ottomane » dans les pratiques musicales des Grecs de Smyrne », Cahiers balkaniques [En ligne], 33 | 2004, mis en ligne le 30 mai 2013, consulté le 06 juillet 2021. URL : http:// journals.openedition.org/ceb/4518; DOI : https://doi.org/10.4000/ceb.4518

Ce document a été généré automatiquement le 6 juillet 2021

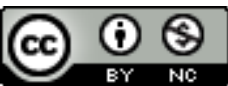

Cahiers balkaniques est mis à disposition selon les termes de la Licence Creative Commons Attribution - Pas d'Utilisation Commerciale 4.0 International. 


\title{
La part « ottomane » dans les pratiques musicales des Grecs de Smyrne
}

\author{
The "Ottoman" part in Smyrnaian Greek musical practice

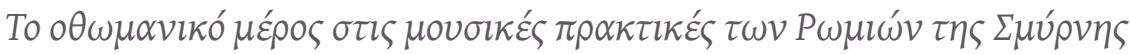

\author{
Basma Zerouali
}

1 À la fin de l'Empire ottoman, Smyrne s'inscrit incontestablement dans la catégorie des grandes cités portuaires cosmopolites de la Méditerranée orientale telles qu'Istanbul, Salonique, Alexandrie ou Beyrouth. Elle se distingue cependant de ces dernières par certains aspects politiques, économiques et démographiques dont les effets conjugués ne manquent pas de se répercuter sur la vie musicale de la ville.

2 Bien qu'elle fasse officiellement partie de l'Empire ottoman jusqu'en mai $1919^{1}$, Smyrne bénéficie dans les faits depuis l'époque byzantine d'un quasi-statut d'extraterritorialité. Sa situation géographique, à la fois au carrefour des routes commerciales et en retrait par rapport au centre du pouvoir politique qu'est Constantinople, lui confère une certaine autonomie, autonomie conservée après la conquête ottomane. Très tôt, dès le $\mathrm{XIII}^{\mathrm{e}}$ siècle, les puissances d'Europe occidentale y jouent un rôle déterminant au plan commercial et économique, mais aussi culturel, rôle qui se renforce au début du XIX ${ }^{e}$ siècle, ce qui vaut alors à Smyrne le surnom de " Paris de l'Orient $»^{2}$.

3 L'autre surnom de la ville, « Gavur Izmir » (Infidèle Smyrne), que lui attribuent les Turcs ottomans, reflète une autre donnée capitale de l'histoire smyrniote entre la fin du XVIII et le début du $\mathrm{xx}^{\mathrm{e}}$ siècle : la prépondérance de la communauté grecque ${ }^{3}$. En effet, durant cette période, l'économie florissante de la ville ${ }^{4}$ provoque un afflux croissant d'immigrés grecs en provenance notamment des îles de l'Égée, du Péloponnèse et de l'Épire, mais aussi de diverses régions d'Asie Mineure, modifiant ainsi le profil démographique de Smyrne au profit de la population grecque, qui devient alors majoritaire et le demeurera jusqu'en 1922. 
4 La première conséquence qu'entraînent ces différentes données sur la vie musicale smyrniote est l'implantation de modèles occidentaux plus tôt, de façon plus marquée et auprès d'un public plus large et plus divers qu'à Istanbul ou dans les autres centres urbains de la région. La raison de ce succès est probablement à rechercher dans le facteur démographique: si la musique occidentale est introduite à Smyrne par les négociants européens, et rapidement adoptée par leurs homologues grecs, ce sont, en fait, les classes moyennes grecques qui semblent assurer sa large diffusion. La présence de ces classes moyennes grecques issues d'horizons géographiques divers, mais en quête d'une identité smyrniote commune, a une autre conséquence, d'une portée encore plus considérable que la première : un métissage entre les musiques pratiquées en Europe occidentale et celles pratiquées en Méditerranée orientale d'une ampleur inégalée à l'époque dans la région.

\section{Étiquette de disque 78 tours}

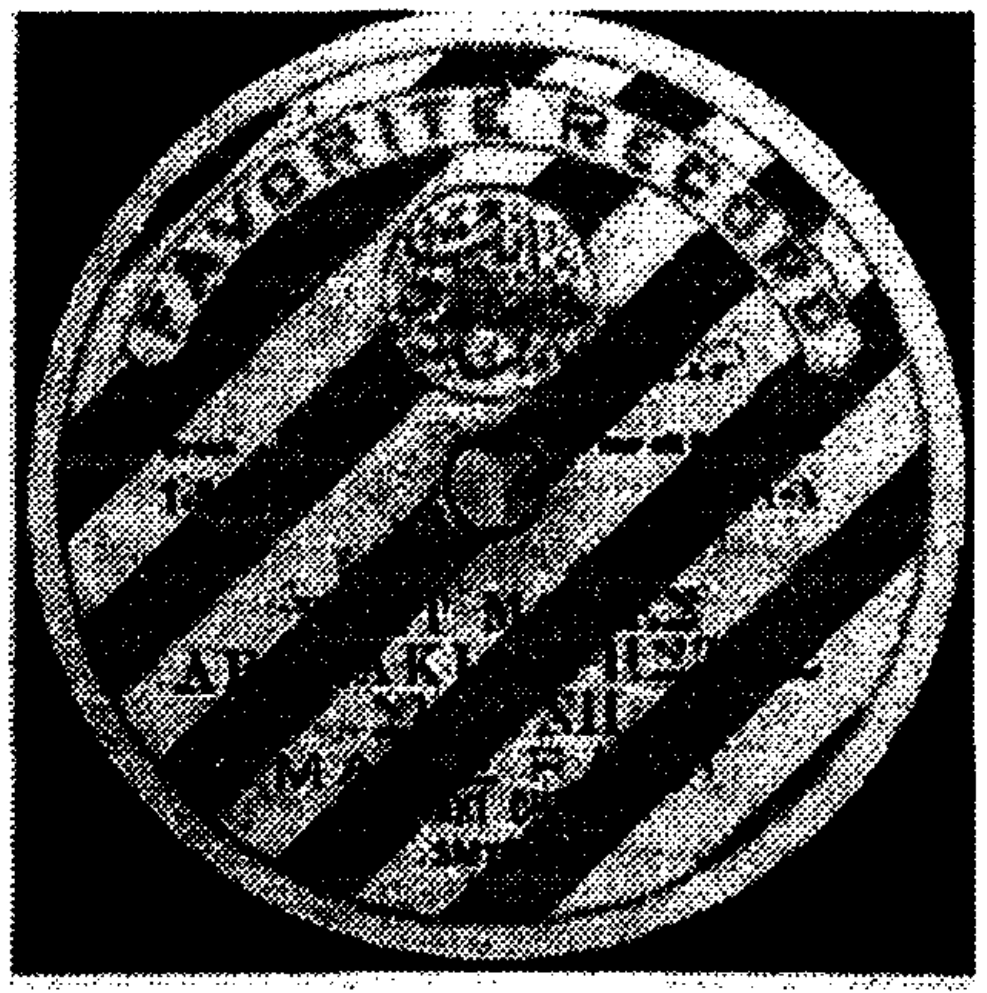

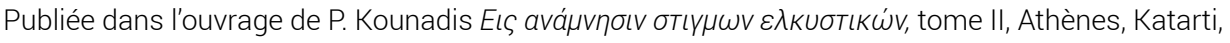
2003, p. 366.

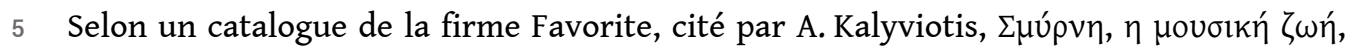
1900-1922, Athènes, Music Corner/Tinella, 2002, p.193), ce Rast manés aurait été enregistré à Smyrne par le chanteur Christos, dit « Arapaki », en 1912.

Dans un tel contexte, les pratiques musicales des Grecs smyrniotes s'inscrivent-elles encore dans un ensemble ottoman ou doivent-elles être considérées à part? Constitueraient-elles, en quelque sorte, une extraterritorialité musicale, analogue au statut de ville franche de Smyrne? 


\section{Musiques « ottomanes »}

7 Avant d'examiner ce que les pratiques musicales des Grecs de Smyrne recèlent d'«ottoman », encore faudrait-il savoir ce que l'on entend par ce terme lorsqu'il est appliqué à la musique. S'il s'agit de l'ensemble des musiques jouées dans l'Empire ottoman, il va de soi que celles des Grecs de Smyrne s'inscrivent d'office dans cet ensemble. Pourtant, rares sont les historiens de la musique et les musicologues qui emploient le terme dans ce premier sens. W. Feldman, l'un des premiers chercheurs à en faire un emploi réitéré à partir du milieu des années 1980, définit la «musique ottomane » comme étant «la musique dominante des régions urbaines de l'Empire ottoman, où le turc était la langue littéraire séculaire de la population musulmane $~^{5}$, incluant par là des genres musicaux variés, mais excluant toutes les provinces arabes et la majeure partie des provinces balkaniques. Le terme n'apparaît en tant que tel dans la discographie qu'au début des années $1990^{6}$, en association avec le répertoire savant.

Carte postale

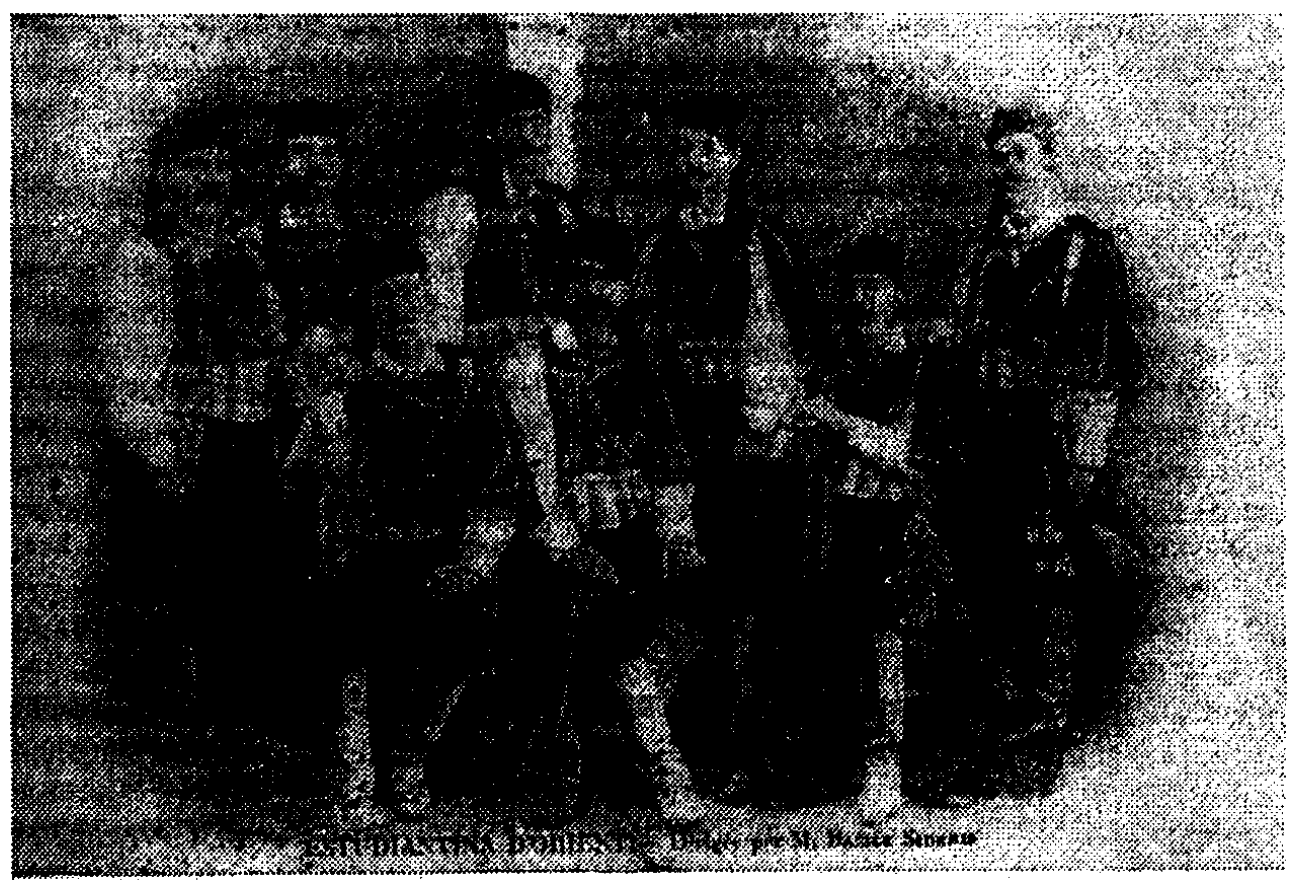

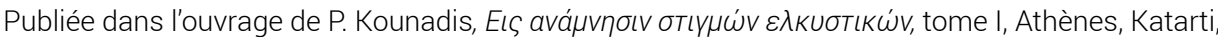
2001, p. 295.

8 Bien que fondée en 1898 à Istanbul, d'où son nom initial de Politakia (les jeunes Stambouliotes), la Smyrneiki Estoudiantina est sans conteste la mandolinata la plus appréciée du public grec "aristocratique» de Smyrne. La cour d'Angleterre suit l'exemple en invitant l'Estoudiantina à participer aux fêtes de couronnement du roi Édouard VII succombe à son tour au charme de cette Estoudiantina.

Dans un article consacré à la « musique juive ottomane », P. J. Dom Sezgin l'emploie en indiquant qu'il correspond à ce que les Turcs modernes appellent klasik Türk müzigi'. Cette dernière expression est effectivement celle utilisée jusqu'à une date récente par l'ensemble des musicologues turcs, ${ }^{8}$ mais aussi par une majorité de musicologues étrangers, dont K. Signell, O. Wright ou U. et K. Reinhardt. Elle ne doit pas sa fortune 
uniquement aux idéologues de la République kémaliste, puisque les sources antérieures aux années 1920 se réfèrent soit à la "musique turque ", soit, plus rarement, à la "musique orientale $"^{9}$ terme qui est certainement trop général, mais qui prend néanmoins en compte le caractère cosmopolite de la musique en question, contrairement au premier. Car peut-on encore de nos jours octroyer un qualificatif ethnique à la musique d'un Empire multiethnique, sachant surtout que nombre de musiciens « ottomans » n'étaient pas « Turcs »?

Réunion sur la place centrale de Pınarbaşı (Bounarbassi)

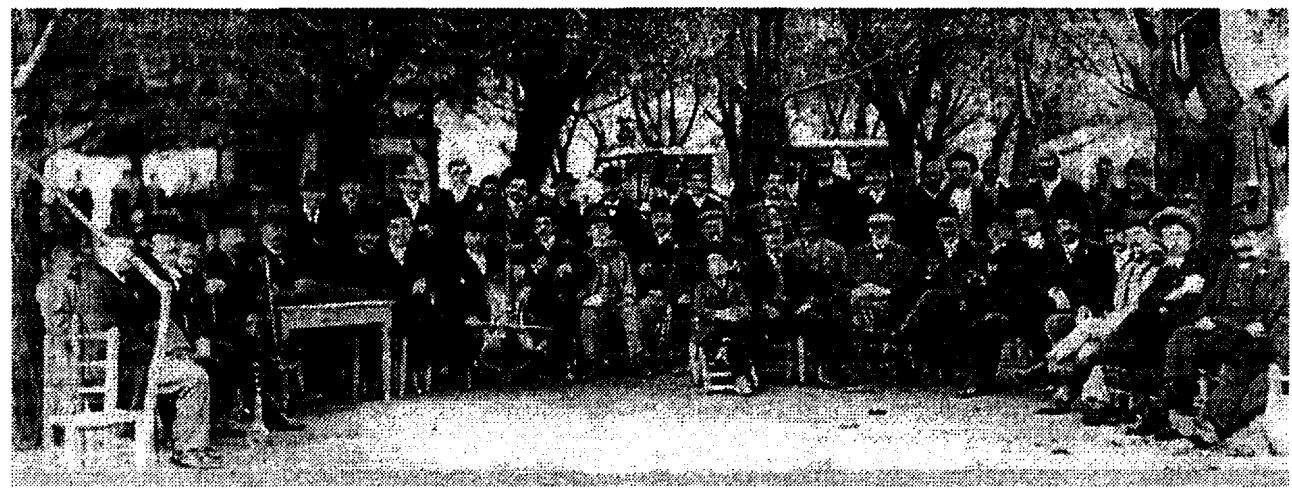

Réalisée dans les environs de Smyrne le 1.12.1900, reproduite en couverture de l'ouvrage de

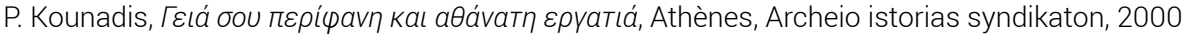

Réunion sur la place centrale de Pınarbaşı (Bounarbassi), lors d'une excursion organisée par la corporation des cordonniers de Smyrne. Au premier plan à gauche

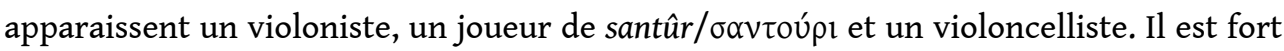
probable que l'homme assis entre ces deux derniers musiciens soit le chanteur.

11 L'emploi même du terme "musique ottomane» semble donc en partie justifié. Cependant, il mériterait d'être étendu à l'ensemble des pratiques musicales partagées par les différentes communautés au sein de l'Empire, et dont la musique savante urbaine ne constitue qu'un aspect: «musiques ottomanes» plutôt que «musique ottomane ». Le cadre de la présente étude ne permettant pas d'aborder le sujet dans son entier, ce qui supposerait de prendre en compte l'ensemble des provinces ottomanes et des communautés y résidant, elle se limitera ici aux rapports entre les pratiques musicales des Grecs de Smyrne et celles des Turcs d'Asie Mineure et d'Istanbul $1^{10}$. Afin d'en simplifier la présentation, ces pratiques seront regroupées en trois catégories, selon le contexte dans lequel elles sont observées : de l'espace sacré au palais; du palais à l'espace urbain; de l'espace urbain à l'espace rural. Il va de soi que ces catégories ne constituent en aucun cas des mondes clos et que les interactions entre elles sont nombreuses.

\section{De l'espace sacré au palais}

12 À la fin de l'Empire ottoman, Smyrne ne semble pas abriter, à la différence d'Istanbul, une école officielle de musique savante séculaire ${ }^{11}$, en dépit du séjour qu'y effectuent des instrumentistes et compositeurs aussi renommés que Tanbûrî Ali Efendi ${ }^{12}$ ou Giriftzen Âsım Bey ${ }^{13}$ et d'un noyau dynamique de musiciens appartenant à la communauté juive, pour la plupart issus des cercles rabbiniques ${ }^{14}$. Car davantage 
encore que dans la capitale, les chantres de la liturgie grecque orthodoxe ${ }^{15}$, les membres des confréries soufies - principalement celle des Mevlevis - et les kantors de la liturgie juive sont à Smyrne les principaux détenteurs de la musique savante.

Si la participation des Grecs d'Istanbul à la vie musicale du palais, de même que les échanges entre chantres orthodoxes du Phanar et soufis sont amplement documentées ${ }^{16}$, les sources ne nous renseignent ni sur la présence de musiciens grecs smyrniotes à la cour des sultans ${ }^{17}$, ni sur les relations que ces derniers peuvent entretenir avec les musiciens des confréries soufies présentes à Smyrne. L'éventualité de tels échanges n'est cependant pas exclue, d'autant plus que les uns et les autres semblent partager nombre de conceptions relatives au "pouvoir mystique de la musique $»^{18}$.

Nous disposons en revanche de plusieurs témoignages datant de la fin du XIX ${ }^{e}$ siècle et du début du $\mathrm{xx}^{\mathrm{e}}$ indiquant la présence de musiciens grecs de Smyrne chez des notables turcs ottomans de la région. Parmi eux se trouvent des chanteurs ayant reçu une formation de chantre, donc parfaitement aptes à interpréter le répertoire savant ottoman. En revanche, aucun musicien grec maitrisant les principaux instruments de ce répertoire que sont le tanbûr ${ }^{19}$ et le ney ${ }^{20} \mathrm{n}^{\prime} \mathrm{y}$ figure ${ }^{21}$.

L'absence de ces deux instruments, plus significative encore que la rareté du 'ûd/ oútı

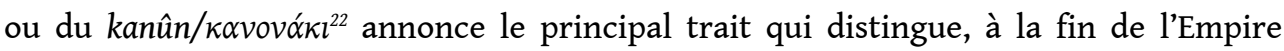
ottoman, les pratiques musicales des Grecs de Smyrne non seulement de celles des Turcs, mais aussi de celles des Grecs de l'Hellade :

\section{L'instrumentarium}

Les Smymiotes pratiquent, certes, la kopsa $(\kappa o ́ \psi \alpha)^{23}$, dérivée du kopuz-i rûmî. Mais cet instrument, tombé en désuétude, ne fait plus partie de l'ensemble de musique savante ottomane depuis le XVI ${ }^{\mathrm{e}}$ siècle. Telle qu'elle est jouée à Smyrne par les Grecs, la kopsa renvoie d'ailleurs à un tout autre contexte. L'absence d'instruments à vent ottomans, qu'ils soient associés à des répertoires savants ou non, est notable, les seuls instruments à vent dont l'usage par les Grecs smyrniotes est confirmé proviennent d'Occident. Des instruments à percussion employés dans la musique savante ottomane,

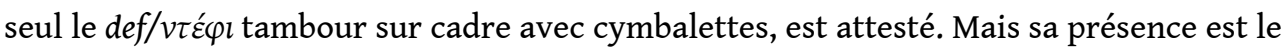
plus souvent constatée lors des fêtes «populaires» ou, parfois, entre les mains des chanteurs se produisant dans les cafés musicaux. 


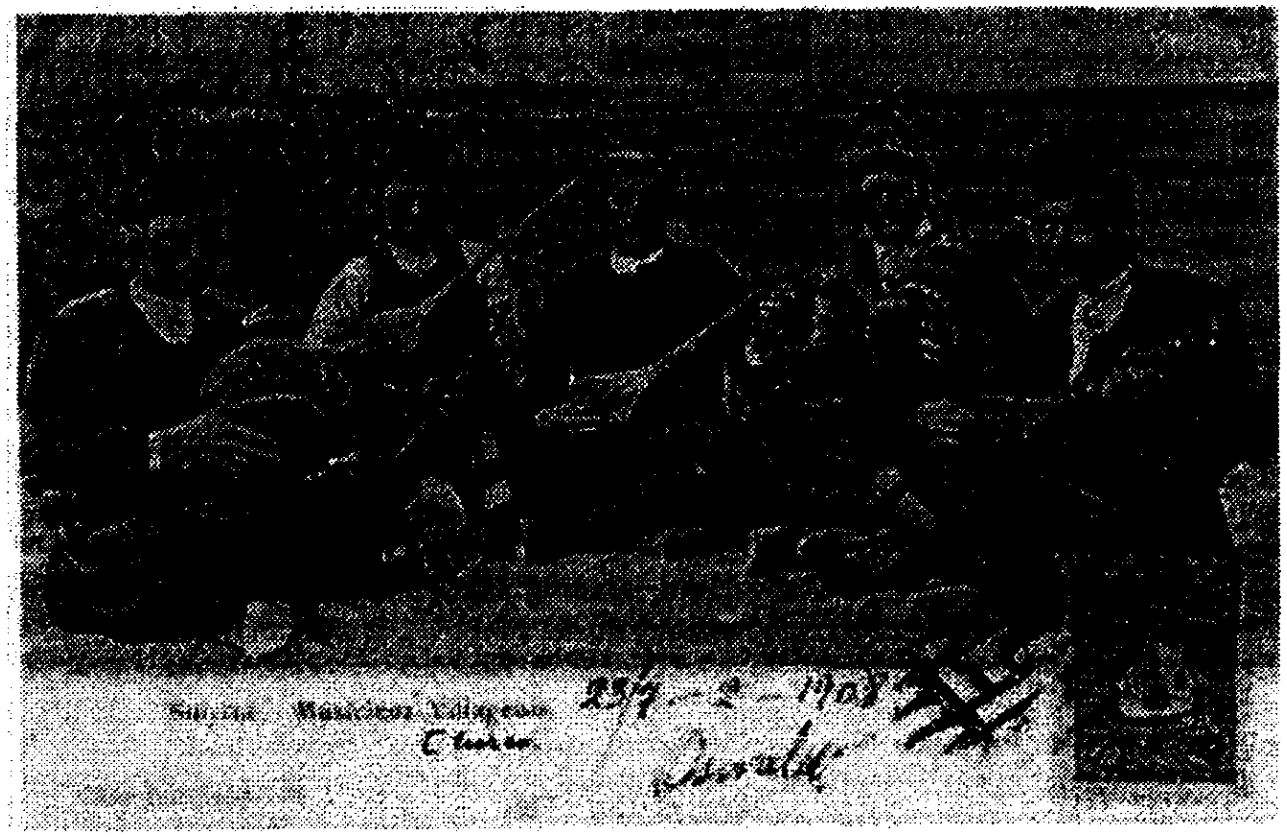

Carte postale datée de février 1908, publiée dans l'ouvrage d'A. Kalyviotis (op. cit.) p. 21.

De gauche à droite, musiciens turcs des environs de Smyrne jouant des instruments suivants dumbelek/toubeleki, baglama/cura, baglama/cura, dumbelek/toubeleki, tambura.

17 Sur le plan de la rythmique, les longs cycles complexes de la musique savante ottomane - qui peuvent compter jusqu'à 88 temps par mesure - sont totalement absents du répertoire grec smyrniote tel qu'il nous est parvenu ${ }^{24}$. Mais ces cycles tendent également à disparaître à la même époque de la musique de l'Enderûn.

En revanche, s'il est une composante de l'idiome musical qui rattache incontestablement les pratiques des Grecs de Smyrne à l'école savante ottomane, c'est bien le matériel mélodique. En effet, en règle générale, le système modal appliqué dans les pièces exécutées par les Grecs de Smyrne n'est autre que celui connu sous la dénomination de makam ${ }^{25}$.

Un examen de la fréquence des makams employés par les Grecs smyrniotes donne des résultats assez proches de celle constatée dans la musique savante ottomane du

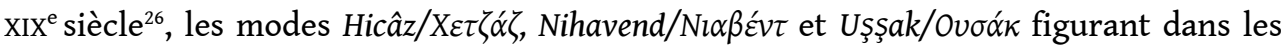
deux cas dans le trio de tête. Les Grecs smyrniotes se distinguent cependant par leur attachement à certains makams prédominants dans la musique savante ottomane à la

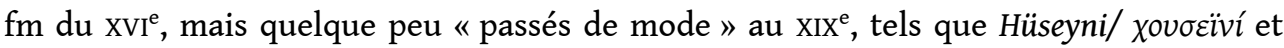
Sabâ/ $\Sigma \alpha \mu \pi \alpha ́ x$, témoignant là d'une ambivalence entre adaptation au changement et " conservatisme» musical ${ }^{27}$.

20 La forme associée à la musique savante ottomane la mieux illustrée par les Grecs de Smyrne, celle du taksîm/ $\tau \alpha \xi i \mu^{28}{ }^{28}$ vocal - désigné depuis le début du xviII siècle par le terme gazel, emprunté à la forme poétique du même nom-constitue un parfait exemple de cette ambivalence. Le gazel ou amané $\left(\alpha \mu \alpha v \varepsilon \varsigma^{29}\right)$ relève selon les Grecs smyrniotes de deux catégories: les "amanédes turcs" et les "amanédes grecs " ${ }^{30}$. La première catégorie ne diffère des gazels turcs contemporains que par l'instrumentation ${ }^{31}$. Elle ne constitue cependant qu'un tiers de l'ensemble des amanédes figurant sur les catalogues discographiques antérieurs à 1922 (ill. 1). Bien que les Grecs 
Smyrniotes n'y demeurent pas insensibles ${ }^{32}$, ils lui préfèrent visiblement la seconde catégorie, qui se distingue notamment de la première par l'absence totale de passages $a$

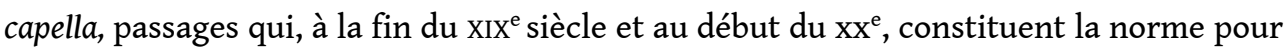
la première catégorie, un ostinato ${ }^{33}$ instrumental n'y apparaissant qu'occasionnellement. Or la description du taksîm vocal tel qu'il est pratiqué à la cour ottomane aux alentours de 1750, fournie par C. Fonton ${ }^{34}$, mentionne clairement cet ostinato. Un demi-siècle plus tôt, D. Kantemiroglu/Cantemir (1673-1723) affirme en outre clairement que les instruments accompagnent mélodiquement le chanteur tout au long de son taksîm, l'ostinato étant alors réservé au taksîm vocal qui clôt la séance musicale ${ }^{35}$. Il semblerait donc que les Grecs de Smyrne demeurent là encore attachés à un trait attesté dans la musique de la cour ottomane à une époque antérieure, dont la conservation devient au $\mathrm{XIX}^{\mathrm{e}}$ siècle une marque distinctive de « leur » amané.

21 Si la première catégorie d'amanédes, à l'image du gazel, requiert des capacités vocales particulières que seuls les chantres de la liturgie byzantine possèdent complètement, la seconde, à l'ambitus plus court et moins mélismatique, est plus accessible aux chanteurs ne bénéficiant pas d'une formation de chantre. D'où la présence de deux styles vocaux parallèles, le premier répondant aux mêmes critères que ceux du gazel nasalisation, intériorisation, trilles - le second caractérisé par une émission plus " naturelle », moins d'ornements et davantage d'expressivité.

Il ne reste étrangement que peu de traces des voix féminines grecques smyrniotes, malgré le renom que certaines d'entre elles ont acquis au-delà de Smyrne. C'est d'ailleurs l'un des articles relatifs à la présence de musiciens smyrniotes dans la capitale de l'État grec, parus dans la presse athénienne durant le dernier quart du $\mathrm{XX}^{\mathrm{e}}$ siècle, qui nous permet aujourd'hui d'imaginer ce que pouvait être l'art de Kior-Katina, la fameuse « Hanende Katingo de Smyrne » ainsi que la présente le journaliste :

Sa voix n'a rien d'extraordinaire, elle est même un peu aigre et désagréablement enrouée, si l'on y prête attention, mais l'art exceptionnel avec lequel elle chante ne nous en laisse pas le loisir. Son chant impose un silence profond, un silence de derviche... ${ }^{36}$

Les rares enregistrements d'A. Papazoglou (1899-1983), bien que réalisés en Grèce au début des années 1930, sont aujourd'hui les seuls témoins irréfutables de l'art vocal des Grecques smyrniotes, la chanteuse ayant accompli la majeure partie de sa carrière avant 1922 à Smyrne ${ }^{37}$. Sa maîtrise autant de la technique savante « ottomane » que de la technique lyrique « occidentale » est impressionnante.

Quant au taksim instrumental, bien qu'il ne figure qu'à titre exceptionnel en tant que "plat de résistance » dans la discographie grecque smyrniote du début du $\mathrm{Xx}^{\mathrm{e}}$ siècle $^{38}$ contrairement à celle des artistes turcs - il est, selon le témoignage de nombreux musiciens, couramment pratiqué.

De toutes les formes vocales composées exécutées à la cour ottomane, seul le sharkı semble être retenu par les Grecs smyrniotes qui mentionnent dans leurs récits plusieurs spécialistes du genre, dont les chanteuses Fikarina de Chios et Anguelara. De cette dernière l'écrivain S. Prokopiou nous dit que « lorsqu'elle interprète des sharkia, les Turcs jettent leurs fez en l'air $»^{39}$. De fait, la structure particulière du shark $1^{40}$ se manifeste dans un grand nombre de pièces chantées par les Grecs de Smyrne.

Il importe cependant de replacer ces formes musicales dans leur contexte de performance, à savoir dans le cycle connu sous le nom de fasll. Les sources se référant directement à la vie musicale smyrniote de la fin $d u x x^{e}$ et du début $d u x^{e}$ du siècle 
restent muettes quant à l'exécution par des musiciens grecs d'un tel cycle à proprement parler. Par ailleurs, les deux principales formes instrumentales composées du fasll, le peşrev et le saz semaisi n'apparaissent pas dans les archives sonores. Cependant, des passages mélodiques empruntés à ces formes, adaptés en tant que chansons ${ }^{41}$, tendraient à indiquer que ces formes sont connues des Grecs smyrniotes. La presse athénienne des années 1875 confirme effectivement que ces formes sont alors pratiquées par ces derniers, dans le cadre de ce qui ressemble fort à un gazino fasil, version "allégée » du fasll de cour, exécutée dans les cafés musicaux d'Istanbul et d'autres centres urbains, dont Smyrne.

\section{Du palais à l'espace urbain}

Bien avant le XIX ${ }^{e}$ siècle, existent déjà à Istanbul certaines institutions ottomanes qui font le lien entre le palais et le monde extérieur, en diffusant un répertoire emprunté à la fois à la musique savante et à des musiques plus "populaires ", musique de danse et chanson urbaine. L'une d'elles est le mehter-i birîm, orchestre composé principalement d'instruments à vent et de percussions, se produisant lors des cérémonies festives, à l'intérieur autant qu'à l'extérieur du palais, afin d'accompagner les danseurs professionnels nommés çengi et koçek. Si les danseuses çengı ${ }^{42}$ sont le plus souvent tsiganes, parmi les köçek ou "garçons danseurs", dont certains se produisent travestis en femmes, se trouvent une majorité de Grecs. Ces danseurs peuvent également être invités à exercer leur talent dans des demeures privées plus modestes que celle du sultan. C'est à ce titre que leur présence est attestée à Smyrne au début du XIX siècle. Un voyageur britannique nous les décrit dans une scène se déroulant à la tombée de la nuit, dans la cour de la maison de l'Aga de Buca, en 1835 :

The Aga was seated on a divan smoking his pipe, regarding with satisfaction the evolutions of the dancers, who elicited occasional loud bursts of merriment from the delighted spectators. The dancers were Greeks, two men, the one in his proper character, the other dressed up as a Turkish female. The scene, I recollect, ended by the man and the pretended woman being seen sprawling together on the floor with the light puffed out, amid peals of laughter from the spectators ${ }^{43}$.

Dans l'Istanbul du xix ${ }^{e}$ siècle, le contexte dans lequel les koçek se produisent le plus fréquemment semble cependant être celui des tavernes et des cafés. Et lorsqu'ils disparaissent de la scène musicale en tant qu'institution, durant le règne d'Abdülaziz, leur répertoire-suites de chansons basées sur les makams, employant le cycle rythmique aksak et se concluant sur un syrtos ou une hor $a^{44}$-leur survit au sein de ces établissements, de même que l'ensemble instrumental qui les y accompagne, évidemment plus restreint que celui du mehter-i birûn, et qui repose essentiellement sur deux instruments à cordes vraisemblablement imposés par les musiciens grecs : la lyra

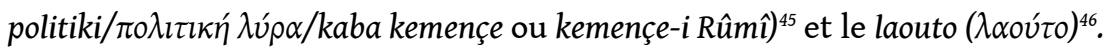

$29 \mathrm{Si}$, tel qu'il nous est parvenu, le répertoire des koçek ne contient aucun élément étranger aux musiciens grecs smyrniotes, il en va, une fois de plus, autrement de l'instrumentarium. Pourtant omniprésent dans les îles de l'Égée, le laouto, pratiqué à Smyrne jusque dans les années 1875 , y tombe en désuétude à la fin $d u$ XIX ${ }^{e}$ siècle tandis que la lyra, qui réussit pourtant à se faire adopter par l'enderûn, n'apparaît qu'au détour d'une rime populaire. Singulièrement, des instruments que les Grecs transmettent à la 
cour d'Istanbul disparaissent du paysage smyrniote ou y sont relégués à un rôle mineur.

La disparition des koçek voit l'émergence parallèle de nouvelles formes de divertissement, dont le tuluat, théâtre improvisé issu de l'orta oyunu mais représenté sur une scène à l'italienne et agrémenté de numéros musicaux désignés par le terme de kanto, forme proche du sharkı, selon C. Poché, quoique plus accessible et « préfigurant la chanson $\aleph^{47}$. Ces numéros sont accompagnés par un ensemble composé d'un trombone, d'une batterie, d'une clarinette et d'un violon, ensemble clairement inspiré des orchestres de "café chantant» européen, mais que les Grecs smyrniotes n'ont apparemment pas retenu. Bien que ces derniers ne le mentionnent pas de façon explicite, le kanto trouve souvent une correspondance dans ce qui sera nommé ultérieurement smyrnéiko tragoudi ${ }^{48}$.

31 Le style vocal des interprètes masculins grecs smyrniotes, similaire à celui des interprètes d'amanédes de la seconde catégorie, est ici également proche de celui des autres interprètes ottomans de kanto. La rareté des documents sonores d'époque concernant les interprètes grecques smyrniotes valant pour tous les genres, la comparaison entre ces chanteuses et leurs consoeurs pourtant nombreuses d'Istanbul peut difficilement être établie ${ }^{49}$.

La présence de musiciens dans les cafés de l'Empire ottoman est documentée depuis l'établissement de ces derniers au $\mathrm{XVI}^{\mathrm{e}}$ siècle. Le $\mathrm{xIX}^{\mathrm{e}}$ siècle voit cependant une importante diversification de leurs activités musicales, due notamment à l'importation des versions européennes du « café chantant » et du « café-concert », qui débouche sur la création d'une nouvelle forme de café musical, désignée dans la capitale ottomane par le terme de gazino et à Smyrne par celui, plus générique, de Kendro. Si à Istanbul, les musiciens grecs partagent la scène de ces établissements avec des musiciens issus d'autres communautés "minoritaires ", à Smyrne, ils l'occupent de manière quasiexclusive, en se distinguant une fois de plus par leurs choix instrumentaux, en l'occurrence celui de la mandolinata (ill. 2) s0 $^{50}$ et, surtout, de l'association violon-santûr/

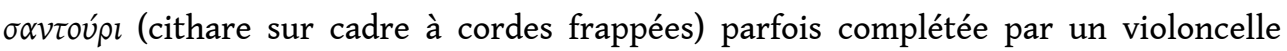
$(\text { ill. 3) })^{51}$.

33 Véritable plaque tournante de la vie musicale smymiote, le kendro n'échappe pas à certains clivages. Ainsi, les établissements «aristocratiques » fréquentés par la haute bourgeoisie grecque de la ville ${ }^{52}$ semblent privilégier les mandolinates et leur répertoire " européen ", tandis qu'à l'autre bout du spectre social, la chanteuse A. Papazoglou évoque de la manière suivante les goûts musicaux des Grecs des "bas-fonds"

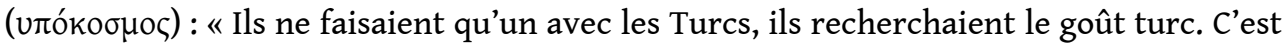
pour ça que celles qui travaillaient dans ces cafés s'habillaient comme des Turques du harem, avec des feredzédes... Ils pensaient ainsi qu'ils étaient des pachas, des aghas, des kaïmakams... Ils n'interprétaient pratiquement que des chants et des danses turques làdedans $»^{53}$.

De tels témoignages doivent évidemment être manipulés avec précaution. Ils constituent en effet des cas extrêmes qui ne doivent pas nous faire oublier ce que révèle un examen approfondi de l'ensemble des sources : dans la Smyrne du XIX siècle, contrairement à Istanbul, les musiques «occidentales» et «orientales» ne se contentent pas de coexister plus ou moins, elles se métissent en transcendant toutes les barrières sociales et ethnico-religieuses ${ }^{54}$. Certaines constituantes de ce métissage 
proviennent de pratiques communes aux Grecs et aux Turcs qui trouvent leur origine hors de l'espace urbain.

\section{De l'espace urbain à l'espace rural}

À Smyrne, il n'est pas rare que les mêmes musiciens qui se produisent dans les cafés musicaux de la ville animent aussi les bals de quartiers et de village, lors de la célébration de fiançailles, de mariages, de baptêmes ou lors des fêtes corporatives (ill.3) et religieuses. Ces dernières en particulier fournissent l'occasion de contacts rapprochés entre les différents millets. Des Turcs musulmans se joignent aux célébrations des grandes fêtes religieuses orthodoxes, comme lors des festivités du carnaval ${ }^{55}$, tandis que des Grecs orthodoxes participent régulièrement aux festivités musulmanes, assistant aux représentations de karagoz et écoutant aussi probablement les meddah et les aşık qui se produisent aux abords du célèbre Pont des Caravanes. Particulièrement significative de ces échanges semble être l'incorporation du héros légendaire de l'épopée turkmène «Koroğlu » aux cérémonies du Carnaval orthodoxe smyrniote sous la forme d'une danse-farandole, le « Kioroglou » ${ }^{56}$.

Le couple d'instruments associé non seulement aux célébrations "villageoises " des Turcs d'Anatolie, mais également aux fêtes rurales des populations helladiques est évidemment celui formé par le zurna et le davul ${ }^{57}$.Or ces instruments sont qualifiés de «musique de Hamit» par la chanteuse A. Papazoglou, qui affirme qu'ils n'étaient employés par les Grecs smyrniotes que lors du Carnaval ${ }^{58}$. Le fait qu'une association instrumentale courante à l'époque et jusqu'à nos jours en Grèce rurale ${ }^{59}$, qui n'est certainement pas la plus fréquemment entendue à la cour du sultan Abdül-Hamid, soit perçue au même moment à Smyrne comme représentative de l'autorité suprême de l'Empire ottoman nous en dit long, d'une part, sur les souvenirs encore vivaces du mehter et, d'autre part, sur l'assimilation par les Grecs de Smyrne d'une identité clairement « citadine ", en dépit des liens étroits de la ville avec son hinterland.

L'emploi des tambours à calice, qui relèvent, eux aussi, de "la musique de Hamit » (ill. 4), semble également être limité aux amateurs et à la période du Carnaval : le Lundi

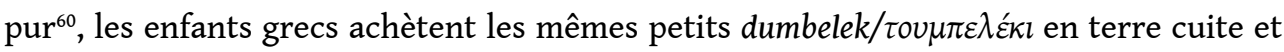
peau de mouton ${ }^{61}$ que les enfants turcs emploient encore aujourd'hui à Smyrne. Pas plus que le zurna ou le davul, le dumbelek n'apparait ni dans les enregistrements d'époque, ni dans les descriptions concernant les musiciens professionnels grecs smyrniotes. 


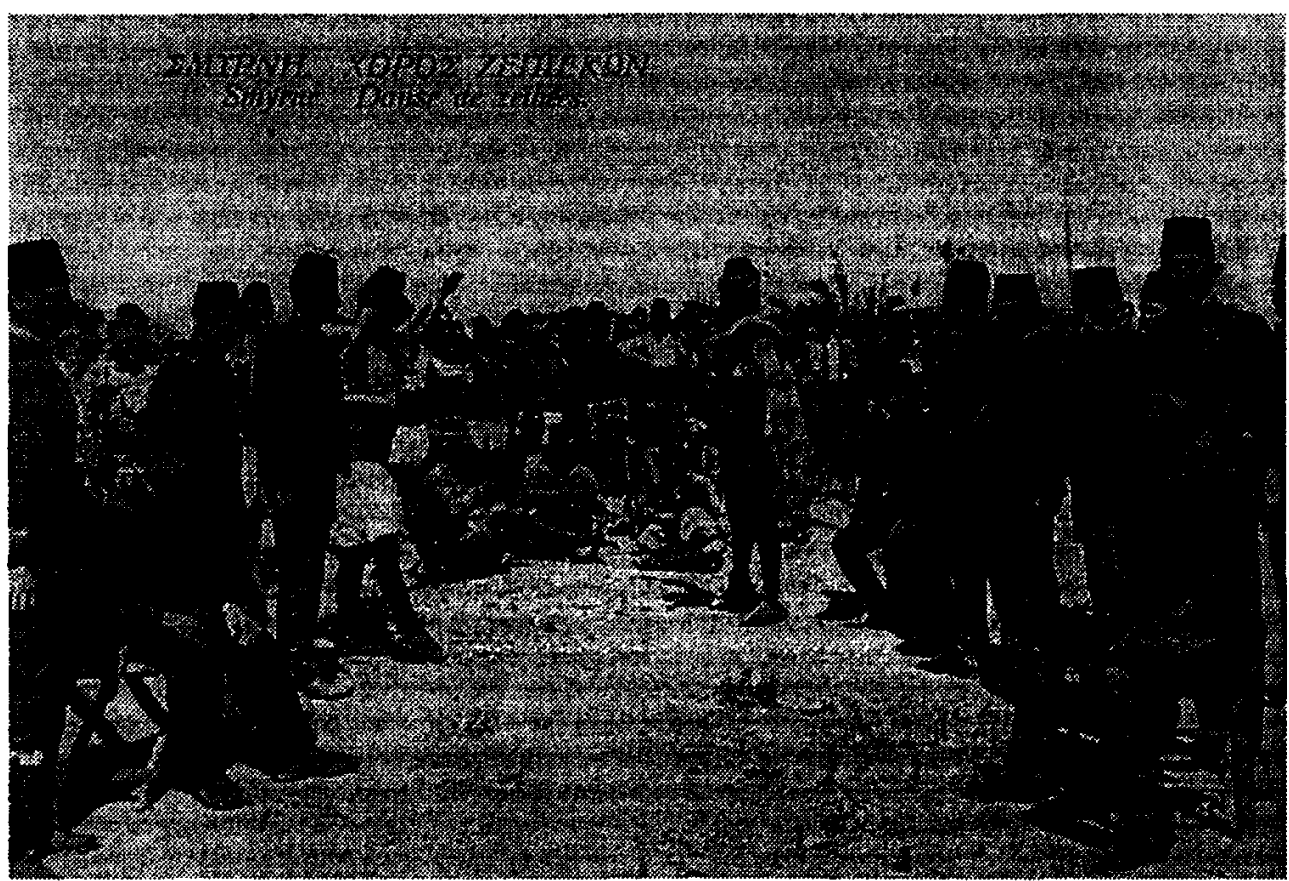

Illustration $n^{\circ} 5$ : carte postale du début du xxe siècle, reproduite dans l'ouvrage $\Sigma \mu u ́ \rho v \eta, \eta \mu \eta т \rho o ́ n o \lambda \eta$

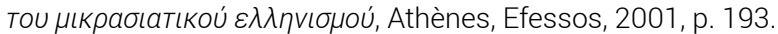

Des Zeybeks (accompagnés par des musiciens dont un semble jouer de l'ikitelli et un autre du dumbelek) dansent en présence d'officiers de l'armée ottomane.

Les luths à long manche, indissociables des pratiques musicales des aşık, sont d'un usage courant parmi les Grecs de nombreuses régions dès l'époque byzantine. En 1842, A. de Valon décrit une jeune fille grecque dans les environs de Buca, jouant d'un instrument de la famille des tambura « La chanteuse reprit d'un ton indolent un éternel récitatif, en s'accompagnant d'un instrument nouveau pour moi. C'était tout simplement un bâton long de deux pieds autour duquel trois cordes étaient tendues. On comprend quelle devait être l'harmonie de cet objet... $»^{62}$. Mais l'emploi de tels instruments par les Grecs smyrniotes semble se restreindre au point de n'être plus évoqués, à la fin du xix ${ }^{\mathrm{e}}$ siècle, que dans un contexte carcéral ${ }^{63}$.

La place dévolue à la danse dans le contexte des séances musicales en plein air explique peut-être le grand nombre de cycles rythmiques que partagent en revanche les Grecs et

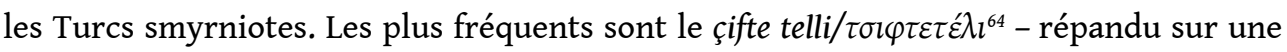
aire qui s'étend du Moyen-Orient aux Balkans et à l'Afrique du Nord et sur lequel s'exécute la fameuse "danse du ventre»- ainsi que deux cycles asymétriques, le

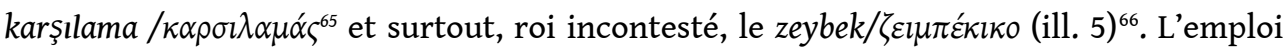
de ces cycles rythmiques fait cependant l'objet d'exploitations différentes selon les cas. À titre d'exemple, le zeybek, tel qu'il est exécuté par les musiciens grecs au début du $\mathrm{xx}^{\mathrm{e}}$ siècle, peut atteindre un tempo extrêmement rapide, inhabituel chez les musiciens turcs de l'époque, d'après les sources discographiques existantes ${ }^{67}$.

\section{Après la tourmente}

Après les événements de 1922, une grande partie du patrimoine musical commun qui vient d'être évoquée sera combattue sur les deux rives de l'Égée: en Grèce, car 
considéré comme l'incarnation des années de soumission aux Turcs et d'un Orient jugé barbare et primitif; dans la jeune République turque en tant que "symbole " du cosmopolitisme décadent ottoman ${ }^{68}$. Mais si la chasse à cette musique indésirable est immédiatement décrétée en Turquie, en Grèce, elle bénéficie d'un sursis jusqu'au milieu des années 1930. Cet entre-temps suffira à assurer la sauvegarde de ce que C. Poché nomme la "quatrième voie de la musique ottomane ${ }^{69}$, qui disparaît avec l'Empire, mais survivra en Grèce grâce aux efforts associés des musiciens smyrniotes et de leurs confrères originaires d'Istanbul.

41 Car dans un apparent paradoxe, les pratiques musicales des Grecs smyrniotes s'«ottomaniseront» de façon radicale après 1922. L'étude comparée des enregistrements réalisés à Smyrne et Istanbul avant 1922 et de ceux réalisés en Grèce entre 1925 et 1935 est à ce titre fort révélatrice ${ }^{70}$. Cette tendance se manifeste également dans le fait que les réfugiés smyrniotes - ainsi que d'autres Micrasiates emploient des mélodies qu'ils partagent avec les Turcs pour relater le drame vécu à la suite de la défaite militaire hellénique en Asie Mineure et afin d'exprimer la douleur du déracinement. Un exemple en particulier retient l'attention. C'est celui d'une marche militaire connue en Turquie comme étant l'œuvre du compositeur Mehmet Ali Bey (1825-1893), sous le titre de "Eski Plevne Marş1 ${ }^{71}$ qui devient un thrène sur l'incendie de Smyrne ${ }^{72}$. Le rébétiko ne saurait enfin être oublié, car c'est essentiellement à travers lui que les pratiques musicales partagées par les Grecs et les Turcs, transmises par les réfugiés, ont fini par s'imposer à la société grecque, en dépit de nombreux obstacles.

Illustration $n^{\circ} 6$ : dessin humoristique de K Mitropoulos, paru le 14.01.2003 dans le quotidien Ta Néa.

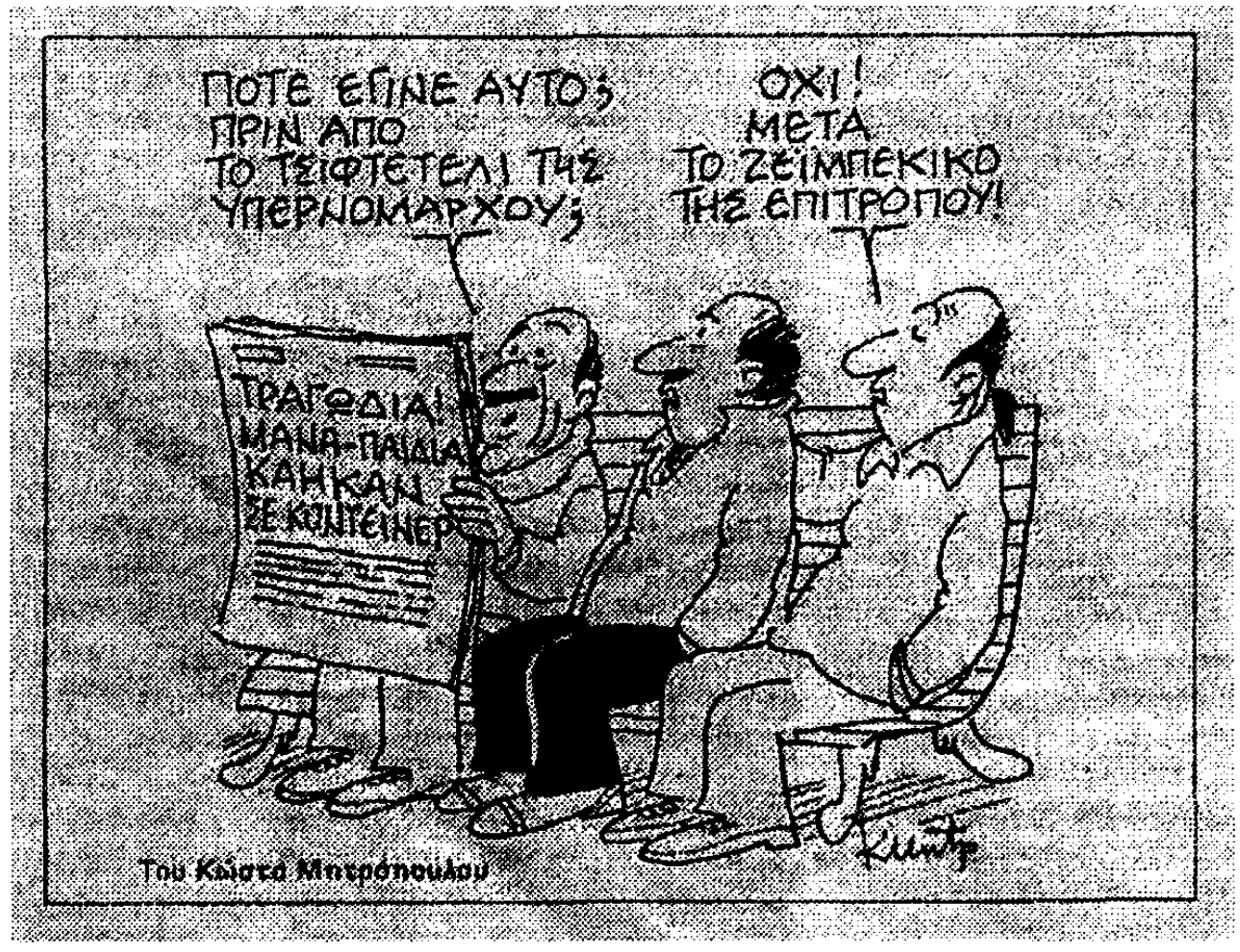

À la lecture de la « une » du journal, intitulée « Tragédie! Une mère et ses enfants brûlés vifs dans un préfabriqué », le premier personnage s'interroge

- Quand est-ce que cela est arrivé ? Avant le tsifteteli du (de la) préfet de région?

- Non! Après le zeybékiko de la commissaire (européenne). 
Sur l'autre rive de l'Égée, les pourfendeurs du patrimoine musical de l'Empire ottoman n'ont pas davantage gagné la bataille puisque ce patrimoine a fini par être réhabilité. Les enregistrements réalisés en Turquie depuis quelques années, notamment par K. Erguner, témoignent en outre d'une nette volonté de revenir aux sources de la musique savante ottomane, à une orchestration plus riche, à des tempi plus lents et à un style plus solennel que celui des enregistrements du début du $\mathrm{xx}^{\mathrm{e}}$ siècle. Étrangement, cette esthétique musicale revisitée influence un bon nombre de productions discographiques grecques consacrées aux musiques de Smyrne depuis le début des années 90 . Les liens entre les styles de la musique liturgique grecque orthodoxe et de la cour ottomane ressurgissent à l'occasion de ces enregistrements, des chantres étant souvent choisis comme interprètes ${ }^{73}$. Signe qu'en dépit des drames historiques, les liens demeurent, générant de nouvelles interactions et un intérêt accru des deux côtés pour un héritage musical commun.

Illustration $n^{\circ} 7$ : couverture du supplément 'Epsilon du quotidien Eleftherotypia, daté du 18.01.2004.

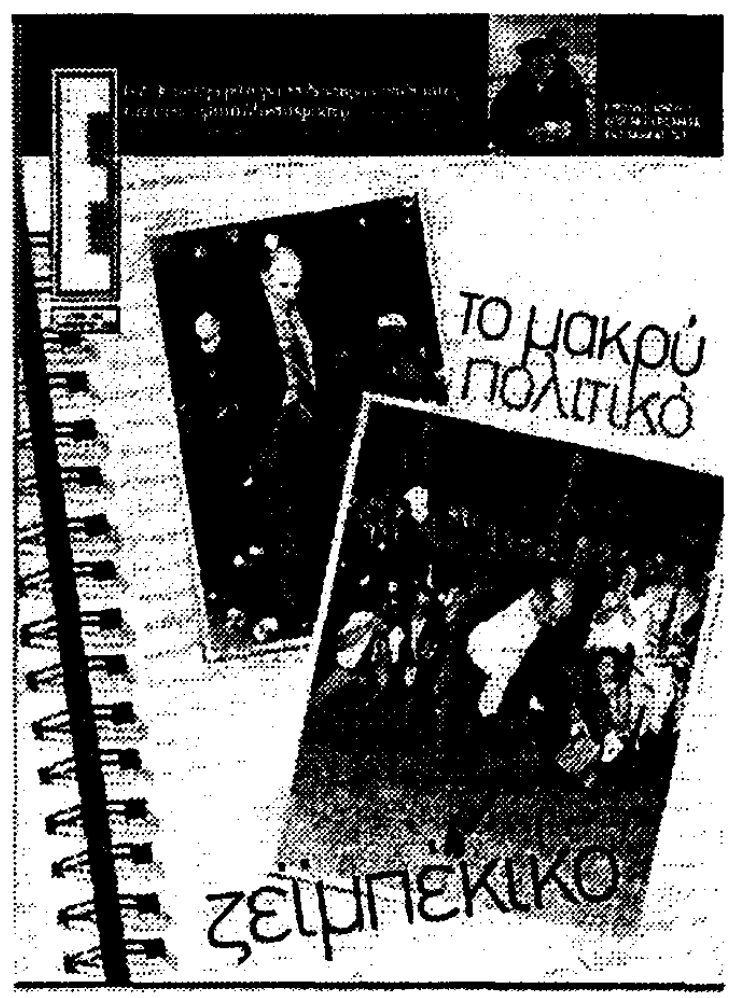

Andreas (lors d'une fête syndicale en janvier 1979) et Georges Papandréou (en juin 2001, sur lî̂le de Samos et en présence d'ïsmail Cem) dansant le « long zeybekiko politique ».

43 Au sein de cet héritage, le zeybékiko occupe assurément une place à part. Il n'a certes pas attendu 1922 pour se faire connaître en Grèce, mais c'est sans conteste aux réfugiés smyrniotes qu'il y doit sa gloire actuelle. Omniprésent dans la musique populaire grecque, le zeybékiko, qui hante les nuits blanches de la jeunesse d'Athènes, du Pirée ou de Salonique, est devenu depuis l'arrivée au pouvoir du parti Pasok, au début des années 1980, un passage quasi obligé dans le parcours public des hommes politiques (ill.6). Lorsque le ministre des Affaires étrangères G. Papandréou rencontre son homologue turc I. Cem sur lî̂le de Samos en juin 2001, il danse... le zeybékiko (ill. 7). Le 25 décembre 2002, le ministre de la Culture E. Venizélos rend visite à des enfants 
tziganes à Ayia Paraskevi, dans la banlieue d'Athènes : il danse... le zeybékiko. Quelques jours plus tard, la préfet de région F. Genimata célèbre sa fête onomastique : elle danse... le zeybékiko. Et même si Mustafa Kemal avait finalement matérialisé son projet constituant à faire du zeybek la danse nationale turque, plus personne aujourd'hui en Grèce ne songerait à contredire A. Papazoglou lorsqu'elle déclarait: «Et maintenant que doit-il se passer? Étant donné qu'ils dansaient le zeybékiko, nous on ne doit pas le danser ? Le zeybékiko est aussi vieux que le monde... $»^{74}$

\section{NOTES}

1. Date du débarquement des troupes hellènes consécutif à la défaite ottomane lors du premier conflit mondial.

2. Selon l'expression de l'académicien J. Michaud (1767-1839), Correspondance d'Orient, vol. 1, Paris, Ducollet, 1833, p. 249.

3. Les termes "grec» et "turc» sont évidemment employés ici dans leur sens ottoman de membres des millets grec orthodoxe et turc musulman, et non pas dans leur sens actuel de ressortissants des deux États-nations correspondants.

4. Économie à laquelle les Grecs contribuent fortement puisque, seuls concurrents sérieux des Occidentaux à Smyrne, ils disposent de réseaux commerciaux s'étendant de l'intérieur de l'Anatolie jusqu'à l'Amérique et au sous-continent indien. Cf. E. Frangakis-Syrett, "The Economie Activities of the Greek Community of Izmir in the Second half of the Nineteenth and Early Twentieth Centuries", Ottoman Greeks in the Age of Nationalism, (dir. C. Issawi \& D. Godivas), Princeton, Darwin Press, 1999, pp. 17-44.

5. “Ottoman Music", New Grave Dictionary of Music and Musicians, 2001, pp. 809-814.

6. Notamment dans les enregistrements réalisés par K. Erguner.

7. "Hakhamim, Dervishes, and Court Singers: The Relationship of Ottoman Jewish Music to Classical Turkish Music", The Jews of the Ottoman Empire (dir. L. Avigdor), Princeton, Darwin Press, 1994, p. 587.

8. Depuis le milieu des années 1990, certains d'entre eux, tels Bülent Aksoy et Cern Behar, emploient également le terme de «musique ottomane ».

9. Tel est le cas de C. Ponton (1725- ?) dans son fameux Essai sur la musique orientale comparée à la musique européenne, manuscrit rédigé en 1751, publié par N. Eckhard, The Science of Music in Islam, vol. 4, Institute for the History of Arabic-Islamic Science at the Johann Wolfgang Goethe University Frankfurt am Main, 1999, pp. 277-340.

10. Les sources prises en compte dans cette étude comprennent, d'une part, des documents sonores-enregistrements d'artistes smyrniotes réalisés à Smyrne et Istanbul avant 1922 et, d'autre part, des récits de voyageurs occidentaux, des articles parus dans la presse smyrniote et athénienne, ainsi que des témoignages recueillis auprès des réfugiés après 1922. Concernant la

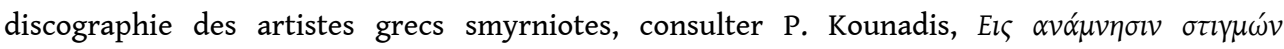
$\varepsilon \lambda \kappa v \sigma \tau i \kappa \omega ́ v$ (En souvenir de moments attrayants), tome I, Athènes, Katarti, 2001 et A. Kalyviotis

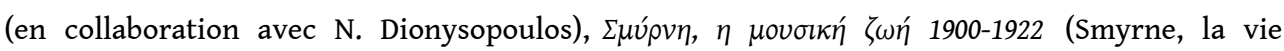
musicale), Athènes, Music Comer/Tinella, 2002.

11. En cela Smyrne, partageant le sort des autres villes provinciales d'Asie Mineure, subit les conséquences de la centralisation de cette musique à la cour ottomane, en particulier après le 
XVII ${ }^{\mathrm{e}}$ siècle. Cf. W. Feldman, Music of the Ottoman Court, Berlin, Verlag für Wissenschaft und Bildung, 1996, pp. 52-54.

12. Né à Mytilène et descendant d'une lignée de hâfiz, Tanbûrî Ali Efendi (1836-1902) exerce l'office de muezzin au Sérail de 1862 à 1885 , date à laquelle il quitte Istanbul pour s'installer à Smyrne où il prodigue son enseignement à Rakım Elkutlu (1872-1948). Selon le musicologue Y. Öztuna (Türk musikisi ansiklopedisi, tome I, Istanbu, 1969), le « romantisme » et le « lyrisme » de Tanbûrî Ali Efendi auraient fortement influencé l'œuvre du légendaire Tanbûrî Cemil Bey (1871-1916).

13. Né à Larissa (Thessalie) et membre de la confrérie des Mevlevis, Âsım Bey (1852-1929) doit à sa maîtrise du girift (flûte oblique en roseau plus petite que le ney) son surnom de Giriftzen. C'est toutefois en tant que joueur de ney qu'il se fait connaître lors de sa résidence à Smyrne entre 1869 et 1872. Pour plus de détails concernant ces musiciens, consulter Y. Öztuna, op.cit.

14. Dont les plus célèbres sont sans doute şemtov şikar (Hoca Santo) ( ?-1920) et Isak al-Gazi (1889-1950).

15. L'un des trois inventeurs de la nouvelle notation musicale "byzantine ", Chtysanthos de Brousse ( ?-1843), Métropolite de Smyrne durant les dernières années de sa vie, lègue à la ville plusieurs disciples.

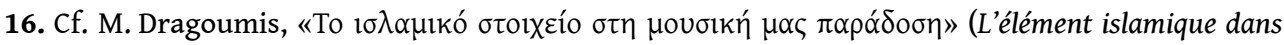

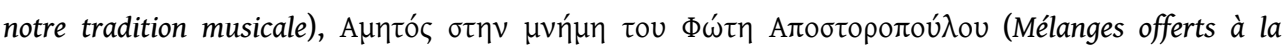
mémoire de Fotis Apostolopoulos), Athènes, Centre d'études d'Asie Mineure, 1984, p. 310-316.

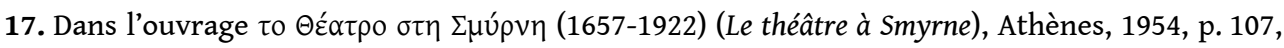
S. Solomonidis évoque la décoration par le sultan Abdül-Hamid du flûtiste, compositeur et chef d'orchestre Victor Kallégias (?-1912), sans toutefois spécifier si ce dernier s'était rendu en personne au palais impérial d'Istanbul. Ce musicien, du reste, semble avoir été davantage familier des marches et opérettes européennes que de l'art du makam.

18. L'expression est empruntée à l'article de J. During intitulé "L'autre oreille, Le pouvoir mystique de la musique au Moyen-Orient ", Cahiers de musiques traditionnelles $\mathrm{n}^{\circ} 3$, "Musique et pouvoirs ", Genève, Ateliers d'ethnomusicologie/AIMP, 1990, p. 55-78.

19. L'instrument le plus complexe de la famille des luths à long manche.

20. Longue flûte en roseau, instrument de prédilection des Mevlevis.

21. La plupart sont violonistes, à l'instar de I. Alexiou, dit Iovanikas, qui, arrêté en 1922, devra la

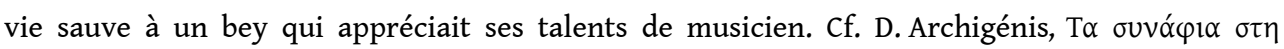
$\Sigma \mu u ́ p v \eta$ (Les corporations de Smyrne), Athènes, 1979, p. 147.

22. Luth à manche court et cithare sur cadre à cordes pincées, instruments peu pratiqués au $\mathrm{XIX}^{\mathrm{e}}$ siècle dans la musique savante ottomane et réintroduits au début $\mathrm{du} \mathrm{Xx}^{\mathrm{e}}$ depuis la Syrie et l'Égypte.

23. Instrument appartenant à la famille des luths à manche court, qui n'est autre que la kobza employée jusqu'à nos jours en Roumanie.

24. Absence à relier à celle des formes reposant sur les cycles longs et complexes.

25. Terme désignant à la fois l'échelle musicale et les règles de la conduite mélodique.

26. Cf. W. Feldman, 1996, p. 235.

27. L'emploi occasionnel par les Grecs de la région de Smyrne d'échelles diatoniques comparables à celles encore pratiquées dans les noubas du Maroc et d'Algérie est peut-être à mettre au compte de ce conservatisme, mais la question dépasse le cadre du présent article.

28. Forme semi-improvisée selon les règles du makam.

29. La dénomination grecque serait dérivée du turc «aman » (pitié), mot répété tout au long de

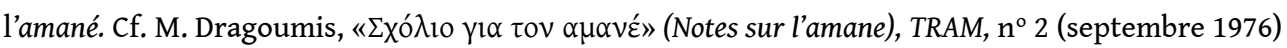
p. 151-157.

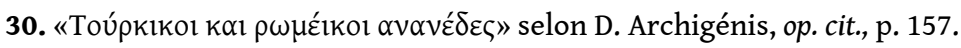


31. Comparer le gazel intitulé Haberin var mı saba kakül-ü (As-tu appris la nouvelle ?), en makam sabâ, enregistré en 1904 pour la firme Zonophone par Hâfiz Mehmet Bey (CD Archives de la musique turque, vol. 1, plage 1) et le Hedzaz manés interprété par I. Tsanakas en 1912 pour le compte de la

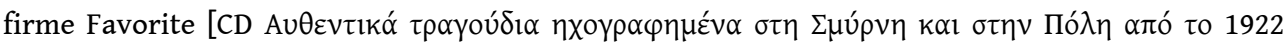
(Chansons authentiques enregistrées à Smyrne et Constantinople avant 1922), plage 14].

32. Selon D. Archigénis (op. cit., p. 157), «Non seulement les Turcs deviennent fous en les entendant, mais aussi les Grecs smyrniotes ».

33. Motif répété de façon continue.

34. Op. cit., p. 311.

35. Cf. W. Feldman, 1996, p. 282.

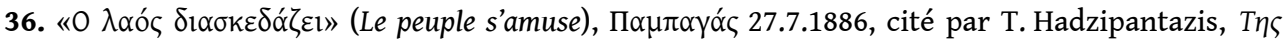

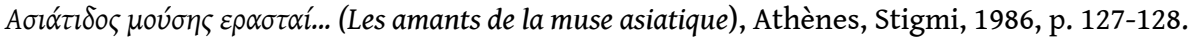

37. L'absence actuelle de données biographiques relatives aux demoiselles Clotilde et Maria ne permet pas de déterminer si ces chanteuses, dont le nom figure sur des étiquettes de disques 78 tours enregistrés à Smyrne en 1909 par la firme Gramophone, sont effectivement Smyrniotes.

38. Un catalogue des disques Gramophone, reproduit par A. Kalyviotis (op.cit., p. 181-184), comporte un Sabah taxim interprété en 1910 au violon par un certain M. Mitsos.

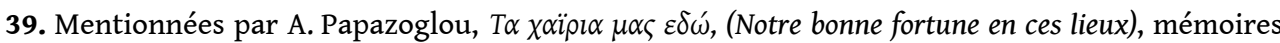

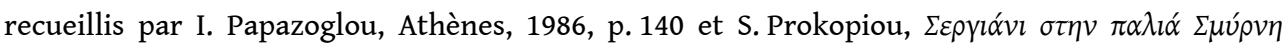
(Promenade dans la vieille Smyrne), Athènes, 1949, p. 166.

40. Constituée de deux lignes mélodiques contrastées, d'une modulation dans la partie médiane, d'un retour à l'une des deux lignes mélodiques du début et d'une ritournelle instrumentale servant lieu de refrain (aranağme).

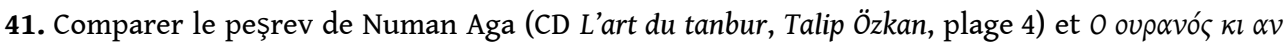

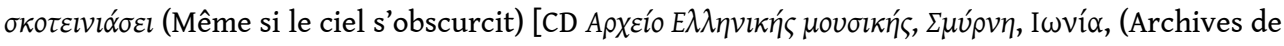
musique grecque, Smyrne, Ionie), plage 9].

42. L'origine de ce terme est vraisemblablement due à la harpe çengı dont jouaient ces danseuses.

43. Récit de C. G. Addison, Damascus and Palmyra, A Journey to the East, vol. 1, Londres, 1838, cité par R Beyru, "Social life in Izmir in the first Half of the 19th century", Three Ages of Izmir, Palimpsest of Cultures, Istanbul, Yapı Kredi Yayımları 1993, p. 206.

44. Cf W. Feldman, 2001, op. cit. L'aksak est dans ce cas précis le cycle asymétrique habituellement transcrit en $9 / 8$. Le syrtos, sur une mesure à 214 , est la danse la plus répandue parmi les populations grecques. La hora, également de nature binaire, est réputée provenir de Roumanie.

45. Vièle d'ongle.

46. Luth à manche court à la caisse moins volumineuse que celle du 'ud et comportant des frettes.

47. Cf. livret accompagnant le CD Archives de la musique turque.

48. Comparer le kanto Ah benim biricigim (Toi, mon unique amour), enregistré en 1905 pour Zonaphone par le Stambouliote Abraham Caracach Effendi (CD Archives de la Musique Turque, vol. 1, plage 16) et le Tsifte telli de l'Elliniki Estoudiantina, enregistré en 1909 à Smyrne pour la firme

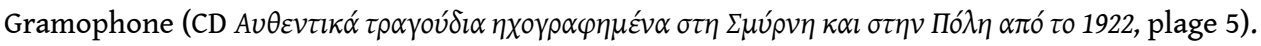

49. Grecques, Arméniennes, Juives et Tsiganes, qui possèdent souvent une voix rauque, de tessiture moyenne ou grave, à l'instar de Victoria Hamm (CD Archives de la Musique Turque, vol. I, plage 6).

50. Ensemble constitué de guitares et de mandolines, également connu sous le nom d'estoudiantina.

51. Association qui fait évidemment penser aux musiciens juifs (klezmorim) et tziganes d'Europe centrale et orientale. Selon W. Feldman (1996, p. 163), le déclin du santûr dans la musique de la cour ottomane serait $d u$ au changement du système d'intonation qui intervient à la fin du XVIII ${ }^{\mathrm{e}}$ siècle. 
52. Ainsi que par la haute bourgeoisie arménienne, juive et levantine.

53. Op. cit., p. 57, 58 .

54. Bien entendu, le kanto a souvent été fourni comme exemple de la pénétration des modèles culturels européens dans l'Istanbul de la fin du XIX ${ }^{\mathrm{e}}$ siècle. Sur un plan strictement musical pourtant, hormis l'instrumentarium, le kanto n'emprunte que peu d'éléments à l'idiome «occidental», contrairement à certaines formes développées par les Grecs de Smyrne. La remarque ne vaut évidemment que pour la période prérépublicaine.

55. S. Prokopiou, op. cit., p. 98.

56. S. Prokopiou, op. cit., p. 98.

57. Le premier est un instrument de la famille des hautbois et le second un tambour à doubleface.

58. A Papazoglou, op. cit., p. 6.

59. Et répandue du reste sur une aire géographique très vaste.

60. Premier jour du Carême orthodoxe.

61. A Papazoglou, op. cit., p. 218.

62. «La Turquie sous Abdul-Medjid", Revue des Deux Mondes, $1^{\text {er }}$ mai 1844, cité par J. C. Berchet, Le voyage en Orient, Paris, Robert Laffont, p. 359, 360.

63. C'est dans ce contexte que S. Prokopiou (op.cit. p. 320) et A Papazoglou (op. cit. p. 53) mentionnent des Grecs jouant du baglama (il s'agit ici du luth à trois cordes appelé cura en Anatolie) et de l'ikitelli (comportant deux cordes, ainsi que son nom l'indique). Toujours selon les mêmes auteurs, il est à noter qu'entre les mains des Turcs et des Arméniens ces deux instruments ne semblent pas avoir la même connotation.

64. Sur une mesure à $4 / 4$ dont les deux premiers temps sont syncopés.

65. Habituellement transcrit en $9 / 8(2+2+2+3)$.

66. Habituellement noté $9 / 4(2+2+2+3$ ou $3+2+2+2)$.

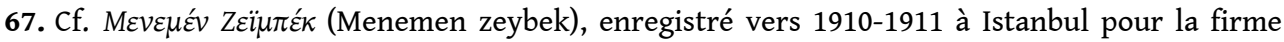

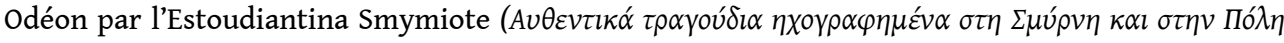

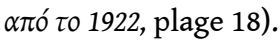

68. Cf. C. Behar, «Ziya Gokalp, le kémalisme et la musique classique turque », Cahiers d'études sur la Méditerranée orientale et le monde turco-iranien, $\mathrm{n}^{\circ}$ 11, Paris, CEMOTI, 1991, p. 9-16 et K. Erguner, «Alla Turca-Alla Franca, les enjeux de la musique turque », Cahiers de musiques traditionnelles $\mathrm{n}^{\circ} 3$, "Musique et pouvoirs », Genève, Ateliers d'ethnomusicologie/AIMP, 1990, pp 45-56.

69. Livret accompagnant le CD Archives de la musique turque.

70. Cf. de l'auteur, "L'amané ou la complainte du réfugié ", Délectations moroses, Paris, L'Harmattan (sous presse).

71. Cf. CD Osmanl Marşlan, The Ottoman Military Music in 78 rpm records, plage 8. La marche est en fait une adaptation d'un hymne du XVI ${ }^{\mathrm{e}}$ siècle, dédié à Osman Paşa (Ancient Turkish Music inEurope, Kecskés Ensemble, plage 7), ainsi que le signale M. Dragoumis à propos de la version

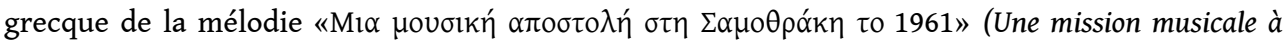

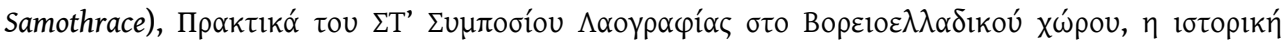

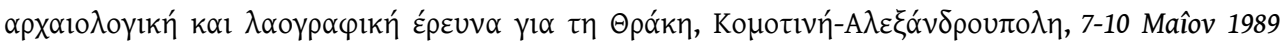
(Actes $\mathrm{du} 6^{\mathrm{e}}$ symposium laographique de la Grèce $\mathrm{du}$ Nord: La recherche historique, archéologique et laographique en Thrace, Komotini-Alexandroupolis, 7-10 mai 1989), Thessalonique, 1991, p. 39].

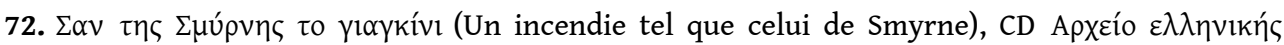

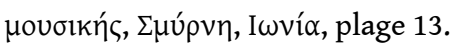

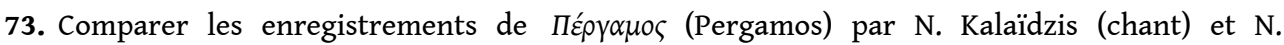

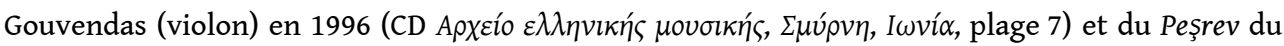


Fasll Hidjaz Humayun par l'Ensemble de Kudsi Erguner en 1990 (Fasll, Musique de l'Empire ottoman, page 1).

74. Op. cit. p. 54.

\section{RÉSUMÉS}

À la fin de l'Empire ottoman, les pratiques musicales des Grecs de Smyrne se distinguent de celles des Turcs par leur caractère incontestablement "métis ", dû notamment à une large adoption d'éléments du langage musical «occidental " et aux particularités d'un instrumentarium où prédominent l'association violon-santûr/sandouri et la mandolinata. Néanmoins, les musiciens grecs smyrniotes continuent jusqu'au début $\mathrm{du} \mathrm{xx}^{\mathrm{e}}$ siècle à partager avec les Turcs ottomans le système modal du makam, certaines formes des répertoires savant et semi savant, ainsi qu'un large éventail de cycles rythmiques, issus des répertoires "populaires", dont certains, à l'instar du zeybek/zeybekiko, sont désormais emblématiques de l'identité culturelle grecque contemporaine.

During the late Ottoman period, the broad incorporation of "Western" musical traits and the predominance of two instrumental ensembles, the one mainly combining violin and santûr/ sandouri and the other one containing guitars and mandolins, distinguish Smymaian Greek musical practices from those of the Turks. Nevertheless, Smymaian Greek musicians continued until the beginning of the 20th century to share with the Ottoman Turks the modal system of the makam, certain parts of the fasil as well as many rhythmic cycles, originating from the "popular" repertoire, some of which, such as the zeybek/zeybekiko, have become emblems of contemporary Greek cultural identity.

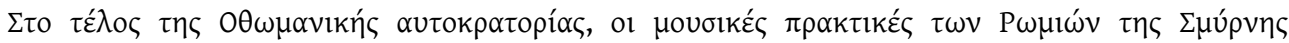

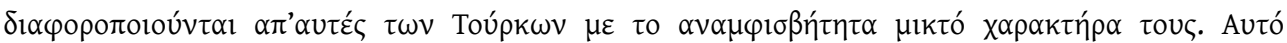

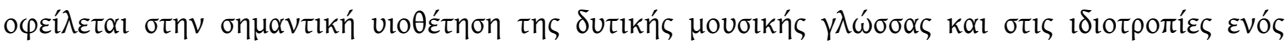

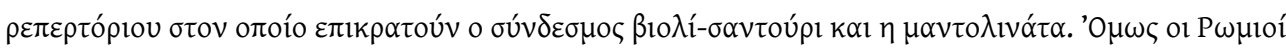

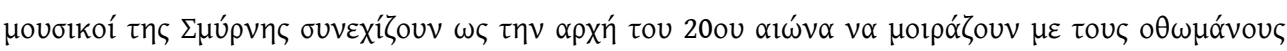

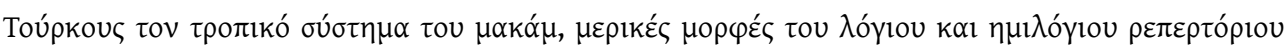

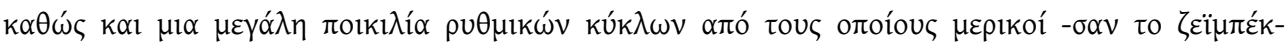

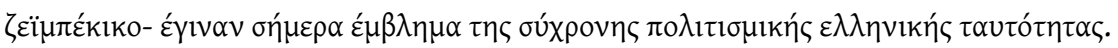

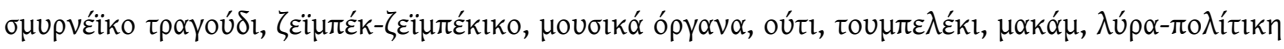

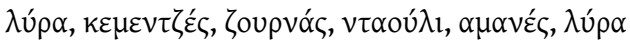


INDEX

Index géographique: Smyrne

motsclesmk РАСПЕАНИ СМИРНА, АМАНЕ, ЖЕБЕКИКО, УД, ДУМБЕЛЕК, ЛИРА, МАКАМ, ТАМБУР, КЕМЕџЕ, ЗУРНА, ДАВУЛ, СМИРНА, ПОЧЕТОКОТ НА ДВАЕСЕТТИОТ ВЕК, АНТРОПОЛОГИЈА

Thèmes: Anthropologie, Musicologie

Mots-clés : chant smyrniote, zeybekiko, zeybekiko, instruments de musique, oud, oud, dumbelek, dumbelek, lyra, lyra, makam, tanbur, amané, zourna, davul, davul

Keywords : Smyrnian songs, Amane, Zeybekiko, music instruments, Ud, Dumbelek, Lyra, Makam, Tanbur, Zurna, Davul, Early twentieth century, Anthropology

motsclestr ızmir'in şarkı, amane, zeybek, ud, dumbelek, lyra, makam, kemence, tanbur ou tanbû, zurna, davul

Index chronologique : vingtième siècle 